\title{
Disengagement of Needle Thread from Rotating Hook, Effects of Its Timing on Tightening Tension, Industrial Single-Needle Lokstitch Sewing Machine
}

\author{
By Yoshinobu Kamata, the late Rikuhiro Kinoshita, Shonosuke Ishikawa* and Kiyoshi Fujisaki**, Members, TMSJ \\ Faculty of Technology, Tokyo University of Agriculture and Technology, Koganei, Tokyo \\ *Present Address: Chori Co., Tokyo Branch, Horidome 2-9, Chuo-ku, Tokyo \\ **Present Address: Newyorker Inc., Soya 548, Hatano-shi, Kanagawa-ken
}

Based on Journal of the Textile Machinery Society of Japan, Transactions, Vol. 35, No. 4, T60-T71 (1982-4)

\begin{abstract}
This paper aims to investigate experimentally the disengagement of needle thread from rotating hook in an industrial single-needle lockstitch sewing machine with respect to tightening tension.

For this purpose, the disengagement of the thread from rotating hook was photographically observed, and the resultant changes in needle thread tension were recorded simultaneously. A rotating hook, manufactured by Hirose Manufacturing Co., Ltd., model HSL-B was used, which was provided with a rotating hook section jib. Sewing speed was $2,250 \mathrm{spm}$, and the sewing threads used were cotton, and filament threads of polyester and nylon.

The experimental investigation above revealed that the timing of the disengagement of needle thread from rotating hook is affected by the elongation properties of the sewing threads.

If the cotton thread low in elongation was used, the needle thread first slips out of the jib and thereafter slips out of the 'rotating hook bobbin case holder position bracket'. This is because when the needle thread becomes tight the rotating hook is beyond the correct angular position. The needle thread behavior therefore is abnormal. In this case, it is estimated that if the shortage of needle thread slack can not be compensated by the thread take-up spring or by thread elongation, the thread is frequently drawn out of the tension disc to result in an insufficient tightening tension.

When polyester or nylon threads higher in elongation than cotton are used disengagement of needle thread from the rotating hook is normal since the needle thread first slips out of the bracket and thereafter slips out of the jib. When the disengagement occurs in this order the timing of disengagement has no effect on the tightening tension.
\end{abstract}

\section{Introduction}

Improper tightening tension of the needle thread creates various problems in sewing with an industrial signle-needle lockstitch sewing machine. If the tightening tension is too large, the stitch becomes too tight to often result in seam puckering and thread breakage. If the tension is too low the stitch becomes loose. Therefore, it is very important to maintain the correct tightening tension.

It is known ${ }^{[1-4]}$ that the tightening tension is affected by various factors such as sewing speed, type of sewing thread, feed lock motion, shape of presser foot, check spring tension, mechanism of thread take-up lever and others. These factors affect tightening tension through such basic mechanisms as the friction coefficients between metals and sewing threads, elongation properties of sewing threads, mechanism of the sewing machine and others. However, the generation of tightening tension is far from clarified either experimentally or theoretically.

According to the experimental study by Ikegami ${ }^{[4]}$ cotton needle thread required higher static tension than polyester needle thread to obtain a proper seam at a sewing speed of 3,600 spm. Consequently tightening tension was higher with cotton thread.

Miyashita, Sekiguchi, Sasahara and Kandachi ${ }^{[1]}$ experimentally studied the effect of sewing speed on the tightening tension. They found that by the use of cotton threads in high speed sewing above 3,000 spm, tightening tension became too low and the stitches were poor in quality. When they increased the needle thread static tension proper stitches were obtained. This is a usual practice in sewing with cotton threads.

As mentioned above, in order to obtain proper stitches in 
sewing with cotton threads, needle thread static tension has to be considerably larger than in polyester filament threads. High static tension, however, can have undesirable effects on sewing threads and also on sewn fabrics because seam puckering may result. It is important, therefore, to have information on how the tightening tension and its variation differ by the type of sewing thread.

Through observation of wave form of needle thread tension, we suspected that the tension of needle thread at the instant of its disengagement from rotating hook affect the tightening tension.

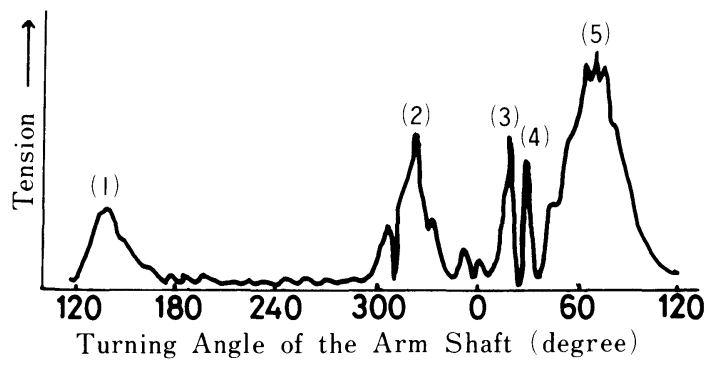

(1) Tension generated when needle penetrates into fabrics.

(2) Tension generated when needle thread goes round rotating hook bobbin case holder.

(3) Tension generated when needle thread slips out of rotating hook section jib.

(4) Tension generated when needle thread slips out of rotating hook bobbin case holder position bracket.

Fig. 1 A typical memory-scope trace of needle thread tension. Sewing conditions: sewing thread-No. 50 cotton; sewing speed-2,250 spm.

In the tension record shown in Figure 1, two peak tension (3) and (4) just before the tightening tension (5) are tension generated when the needle thread slips out of the rotating hook section jib and the 'rotating hook bobbin case holder position bracket' (hereafter the former is called the jib slipping tension, and the latter is called the bracket slipping tension, and both tension combined is called the hook slipping tension). In view of above it can be speculated that if these tension are too large extra needle thread is drawn out of the tension disc to interfere with the tightening action of the take-up lever.

In the present study the process of the disengagement of needle threads from the rotating hook were photographically observed, and the needle thread tension was recorded simultaneously to clarify the cause and effect relation between the two.

\section{Sewing Conditions}

Sewing conditions are shown in Table 1. Cotton threads and filament threads of polyester and nylon were compared keeping other conditions identical. The bobbin thread static tension of $\mathbf{3 0} \mathbf{g f}$ and the needle thread static tension of $\mathbf{7 0} \mathbf{g f}$ were chosen in order to obtain good seams in sewing with polyester filament threads. The tightening ratio was $91 \%$ with polyester threads, $94 \%$ with the nylon threads and $118 \%$ with the cotton threads. The stitches in sewing with cotton threads were too loose and unsatisfactory.

Table 1 Sewing conditions.

1. Sewing Machine-Janome DB-J 704 industrial single-needle lockstitch sewing machine.

Needle thread static tension: $70 \mathrm{gf}$

Bobbin thread static tension: $\mathbf{3 0} \mathrm{gf}$

Pressure of presser foot: $4 \mathrm{kgf}$

Check spring tension: $50 \mathrm{gf}$

Stitch dial number: 2.5

Sewing speed: $2,250 \mathrm{spm}$

2. Fabric-T/C broadcloth ( 2 ply)

3. Needle-DB $\times 1$ No. 11

4. Sewing Threads-No. 50 cotton

No. 50 polyester filament

No. 50 nylon filament

The tightening ratio above is the ratio of needle thread length to bobbin thread length in a given seam length.

A rotating hook, model HSL-B, which is manufactured by Hirose Manufacturing Co., Ltd. used in the present study is shown photographically in Figure 2 since the shape of the hook is important. The hook is for general use characterized by a long rotating hook section jib.

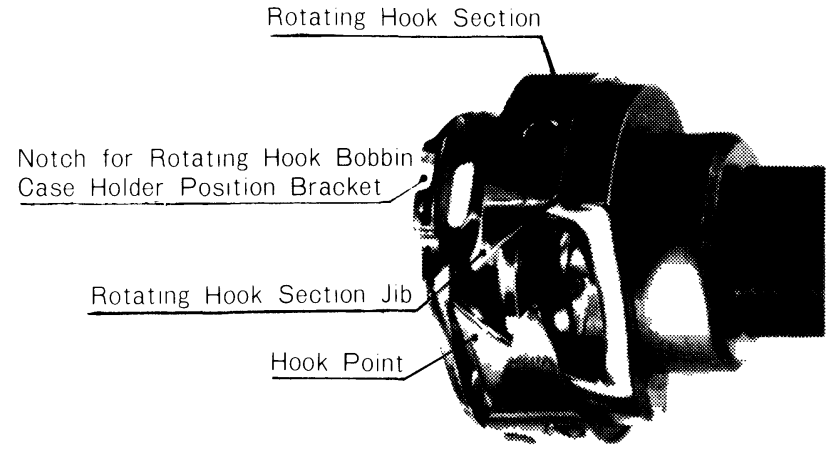

Fig. 2 A rotating hook, model HSL-B (Hirose Manufacturing Co., Ltd.), used in this work. 


\section{Experimental}

\subsection{Observation of Needle Thread Behavior in the Vicinity of Rotating Hook}

Figure 3 illustrates the observation of needle thread be-

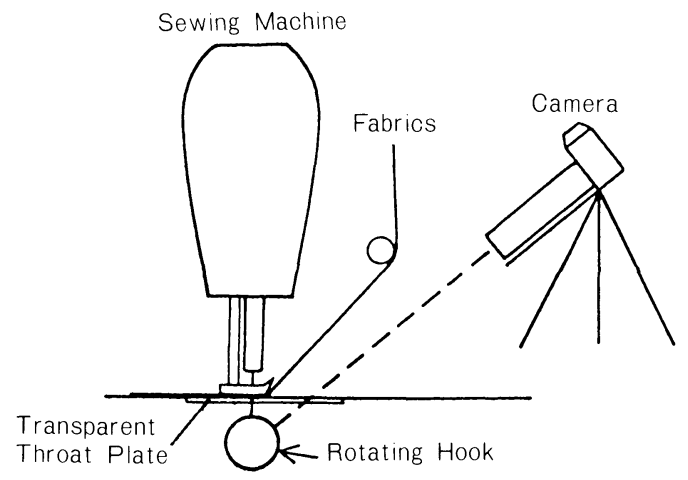

Fig. 3 Schematic of the observation of needle thread in sewing.

havior in the vicinity of rotating hook. To facilitate observation, entering fabric were deflected upward, a throat plate made of a transparent acrylic plate $3 \mathrm{~mm}$ thick was provided, and the front end of the pressor foot was cut by a length of about $9 \mathrm{~mm}$.

\subsection{Measurement of Needle Thread Tension}

Following the usual practice a detector for measuring needle thread tension was installed between the thread takeup lever and the needle as shown photographically in Figure 4. The needle thread tension is detected by sensing the de-

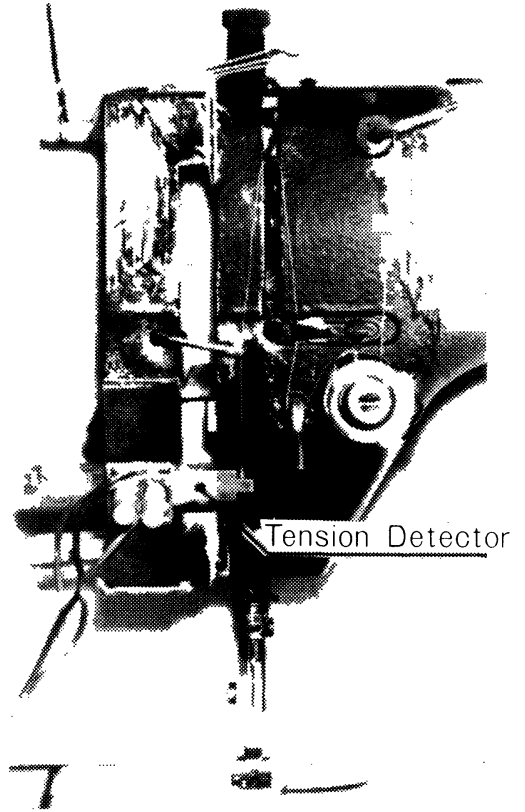

Fig. 4 Photograph of needle thread tension detector. flection of a cantilever beam using semi-conductor strain gauges. The natural frequency of the cantilever beam was about $9 \mathrm{kHz}$. The signal from the sensor was displayed and recorded on a memori-scope by way of a dynamic strain amplifier.

\subsection{Simultaneous Measurement of Needle Thread Tension and Needle Thread Movement}

Shown in Figure 5 is the system which observes photographically the movement of the needle thread slipping out

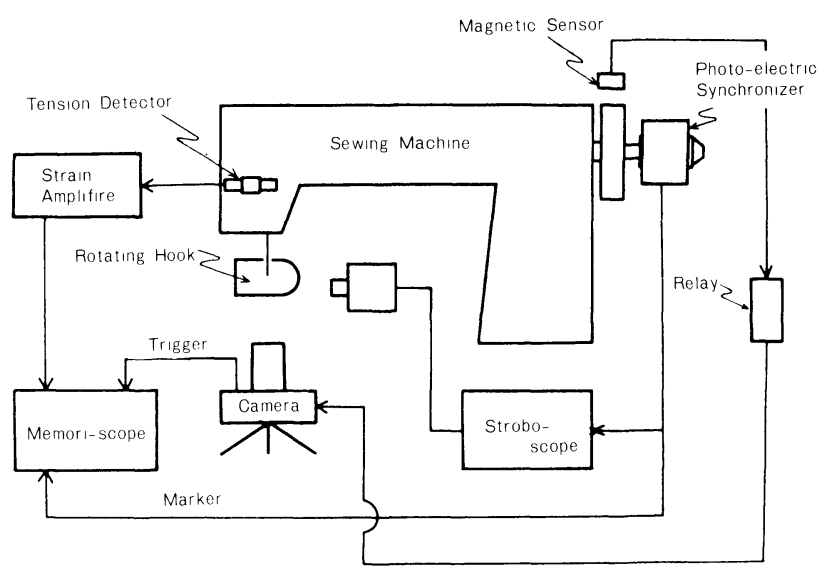

Fig. 5 Block diagram of measuring system.

of the rotating hook and at the same time records the thread tension generated. A photo-electric synchronizer placed at the arm shaft generates a synchronized signal indicating a particular turning angle of the arm shaft. The signal triggers strobo-scope flash for the photographical recording of thread shape at that arm shaft angle. Also, the signal enters the memory-scope to provide a time marker. Thus, the time instant of photography shot is marked on the memori-scope thread tension record. To further insure the synchronization of the camera to the memori-scope, the motor drive of the camera is controlled by signals from magnetic sensor independently installed on the arm shaft. The $\mathrm{X}$ contact point of the camera is used as an external trigger for the memori-scope to enable a simultaneous measurement of both the needle thread tension and the needle thread shape.

A typical example of the measurement with polyester filament threads is shown in Figure 6. Needle thread tension record is shown in the lower part of the figure and the needle thread profile at the time instant indicated by the marker is shown photographically in the upper part. Evidently the jib and the bracket hold the needle thread tightely.

Since sewing speed in the experiment was $2,250 \mathrm{spm}$ making the speed of rotating hook 4,500 rpm and the outer diameter of the hook was about $34 \mathrm{~mm}$, the rotating hook turned by an angle of 0.54 degrees and the outer surface move by a distance of $0.16 \mathrm{~mm}$ during the strobo-scope 

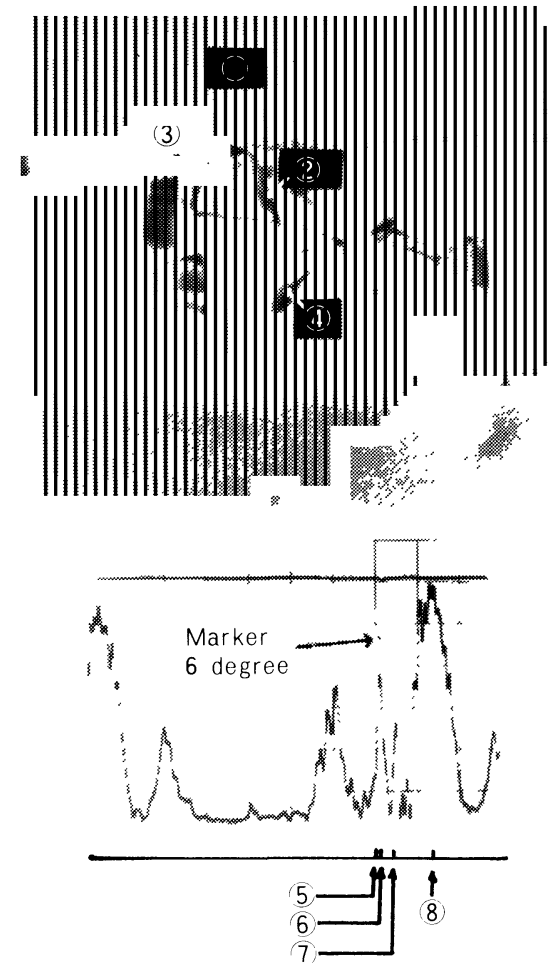

(1) Fabric

(2) Needle thread

(3) Rotating hook bobbin case holder position bracket

(4) Rotating hook section jib

(5) Upper dead point of needle bar ( 0 degrees)

(6) Bracket slipping tension

(7) Jib slipping tension

(8) Tightening tension

Sewing conditions: sewing thread-No. 50 polyester filament thread; sewing speed-2,250 spm.

Fig. 6 An example of simultaneous measurement of needle thread tension and needle thread profile.

flash duration of $20 \mu \mathrm{s}$. As Fig. 6 shows this movement evidently did not blur.

\section{Experimental Results}

\subsection{Correlation between Tightening Tension and Hook} Slipping Tension

Figures 7, 8, and 9 compare polyester, nylon and cotton in terms of the correlation between the tightening tension and the hook slipping tension which later is the needle thread tension at the moment of its disengagement from the jib or the bracket.

Taken in abscissa is the B tensions which is defined as the

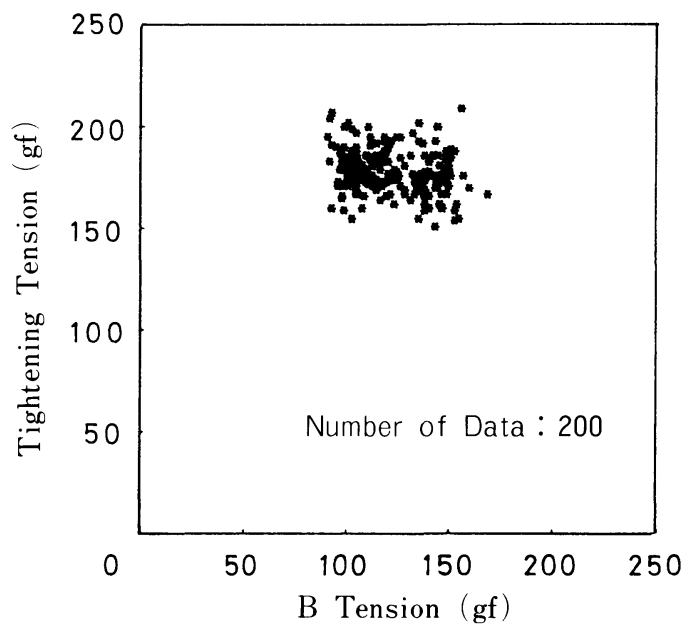

Fig. 7 Correlation between hook slipping tension and tightening tension with No. 50 polyester filament sewing thread.

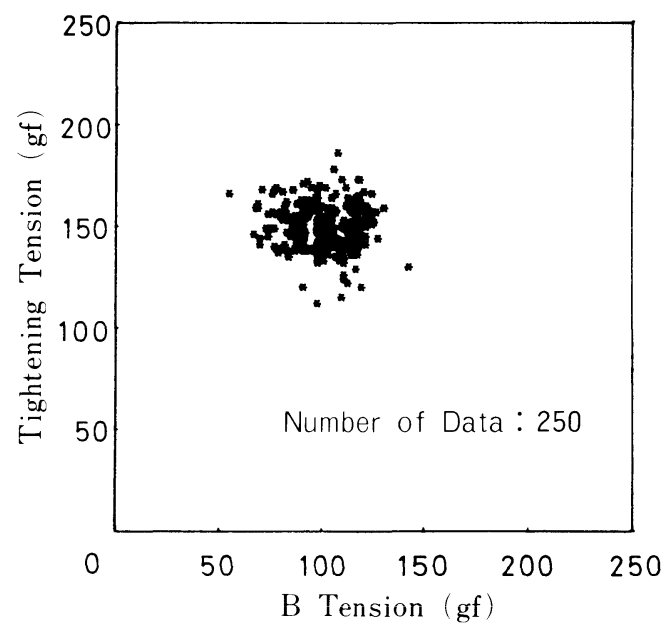

Fig. 8 Correlation between hook slipping tension and tightening tension with No. 50 nylon filament sewing thread.

larger of the jib slipping tension and the bracket slipping tension. From these figures, it was found that, in sewing with polyester or nylon threads, the correlation between tightening tension and $\mathrm{B}$ tension was not significant, and both the tightening tension and the $\mathrm{B}$ tension fall within a narrow range. In the case of the cotton threads, however, a significant correlation between the two tension was observed (the correlation coefficient: -0.56 , data number: 200$)$. In sewing with cotton threads, the tightening tension decreases with increasing B tension or hook slipping tension and the scatter of tightening tension increases with increasing $\mathrm{B}$ tension. 


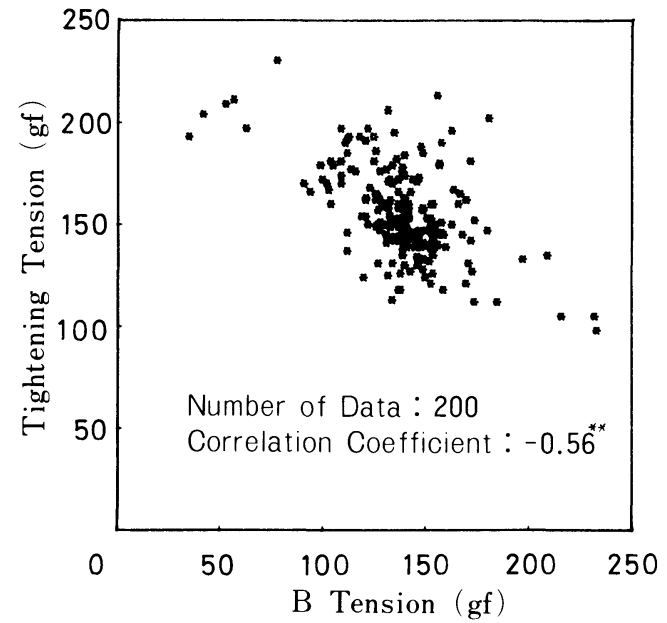

Fig. 9 Correlation between hook slipping tension and tightening tension with No. 50 cotton sewing thread.

In view of above the rest of this paper aims mainly to clarify the reason why the hook slipping tension affects the tightening tension when unsuitable sewing threads such as cotton threads are used.

\subsection{Disengagement of Needle Threads from Rotating Hook and its Classification}

Peak tensions generated when needle threads slips out of the rotating hook are the bracket slipping tension and the jib slipping tension. These tension, however, vary widely in their peak magnitude and in the location of the peak in arm shaft angle. To clarify the cause and effect relation between thread profile and thread tension large numbers of thread profile pictures and thread tension records as the ones shown in Fig. 6 were taken under different sewing conditions and classified into the types listed in Table 2. Table 2 reveals the following.

(1) Polyester and nylon threads were very similar in the manner of disengagement from rotating hook, but cotton threads were distinctively different.

(2) Figures 10 through 12 show thread tension records and thread profiles at the time instant indicated by the marker dot. Figures 10 and 11 show type $C$, polyester thread, and Figures 12 and 13 show type $C^{\prime}$, cotton thread. Pictures for nylon threads are not shown due to the similarity to polyester threads.

(3) Precise nature of the two tension peaks generated when the needle thread slips out of the hook has so far been an object of guesswork since tension record was the only in-

Table 2. Classification of the various types of hook slipping tension

\begin{tabular}{|c|c|c|c|c|c|c|c|c|c|}
\hline \multicolumn{2}{|c|}{ Sewing thread } & \multicolumn{2}{|l|}{ Polyester } & \multicolumn{2}{|l|}{ Nylon } & & & \multicolumn{2}{|l|}{ Cotton } \\
\hline & & \multicolumn{4}{|c|}{ Turning angle of the arm shaft (degrees) } & & & \multicolumn{2}{|c|}{$\begin{array}{l}\text { Turning angle of the } \\
\text { arm shaft (degrees) }\end{array}$} \\
\hline \multicolumn{2}{|c|}{ Type } & \begin{tabular}{|l|} 
Slipped \\
out of the \\
bracket
\end{tabular} & \begin{tabular}{|l|} 
Slipped \\
out of the \\
jib
\end{tabular} & \begin{tabular}{|l|} 
Slipped \\
out of the \\
bracket
\end{tabular} & \begin{tabular}{|l|} 
Slipped \\
out of the \\
jib
\end{tabular} & \multicolumn{2}{|c|}{ Type } & \multirow{2}{*}{$\begin{array}{l}\text { Slipped out } \\
\text { of the } \\
\text { bracket } \\
27-28 \\
\end{array}$} & \multirow{2}{*}{$\begin{array}{l}\begin{array}{l}\text { Slipped out } \\
\text { of the jib }\end{array} \\
19\end{array}$} \\
\hline A & $\stackrel{a}{a}$ & -4 & $21-22$ & -2 & $21-22$ & $\mathbf{A}^{\prime}$ & $\stackrel{h}{a}^{a}$ & & \\
\hline B & $M$ & $6-7$ & $21-22$ & $5-6$ & $21-22$ & $\mathbf{B}^{\prime}$ & $\stackrel{M}{a}^{a}$ & $30-31$ & 19 \\
\hline \multirow[t]{2}{*}{$\mathrm{C}$} & $M^{b}$ & $9-10$ & $21-22$ & $8-9$ & $21-22$ & $\mathbf{C}^{\prime}$ & $\stackrel{b}{N}$ & $32-33$ & 19 \\
\hline & & & & & & $\mathbf{D}^{\prime}$ & $\stackrel{a b}{M}$ & under 15 & $20-21$ \\
\hline
\end{tabular}

A type: The bracket slipping tension, a, generates very small, and the jib slipping tension, $b$, generates very large.

B type: The jib slipping tension, $b$, generates large than the bracket slipping tension, $a$.

C type: This is contrary to $B$ type.

$A^{\prime}$ type: This is the same as $A$ type, however the tensions generates in the reverse order.

$B^{\prime}$ type: This is the same as B type, however the tensions generates in the reverse order.

$\mathrm{C}^{\prime}$ type: This is the same as $\mathrm{C}$ type, however the tensions generates in the reverse order and the bracket slipping tension, a, generates abnormally large.

$\mathrm{D}^{\prime}$ type: Both tensions generate very little. 

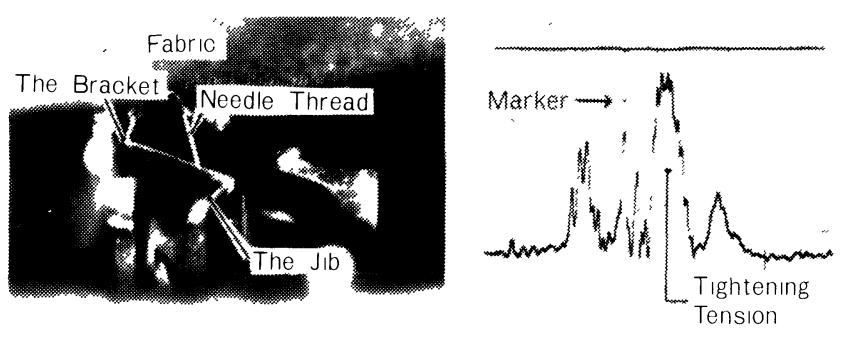

(a) 8 degrees
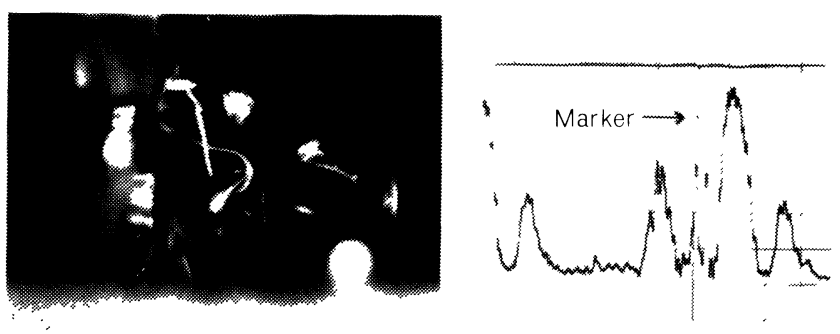

(b) 9 degrees
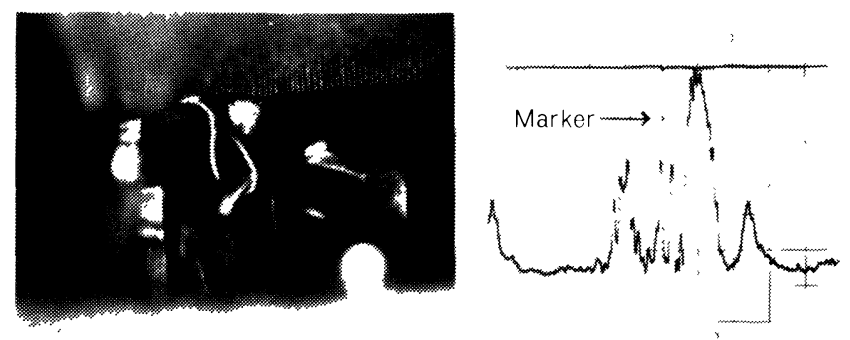

(c) 10 degrees

Fig. 10 Typical photographs of polyester needle threads slipping out of the bracket (C type).

formation available. It was clarified by the present simultaneous observation of needle thread tension and needle thread profile. With polyester or nylon threads the needle thread first slips out of the bracket and then out of the jib. On the other hand, with cotton threads, the needle thread first slips out of the jib and then out of the bracket. In other words, the order of thread disengagement differs by the sewing thread used.

However, with cotton threads there is type $\mathbf{D}^{\prime}$ in which practically no hook slipping tension was observed. In this case, cotton threads were simular to polyester threads in behavior. This can be considered an exception since its probability of appearance is only $2 \%$.

(4) The turning angle of the arm shaft when the thread slips out of the jib was from 21 to 22 degrees with polyester or nylon threads, while it was 18 degrees with cotton threads. The difference between the two was small and the disengage-
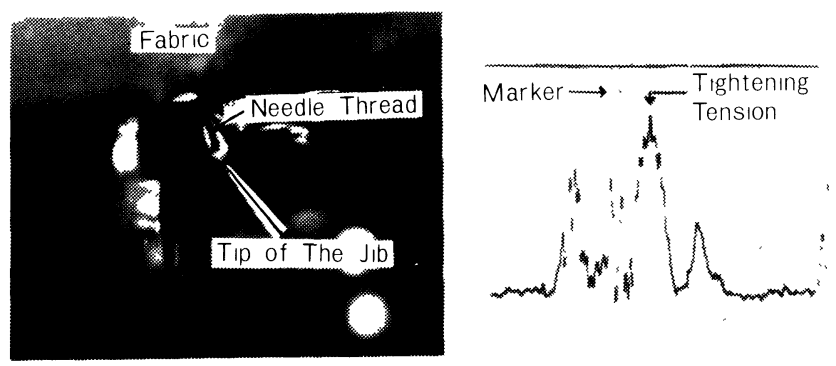

(a) 22 degrees
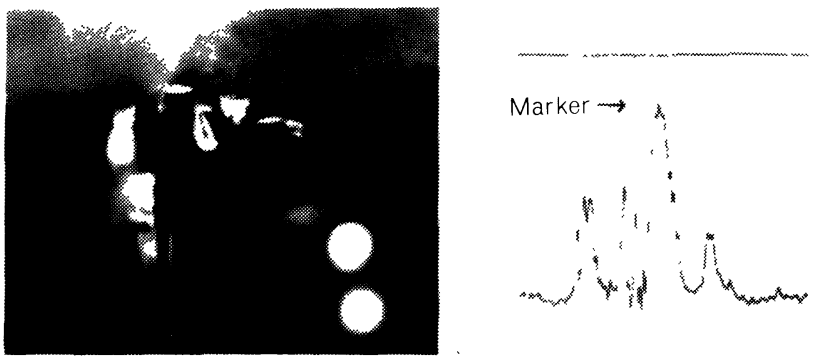

(b) 23 degrees
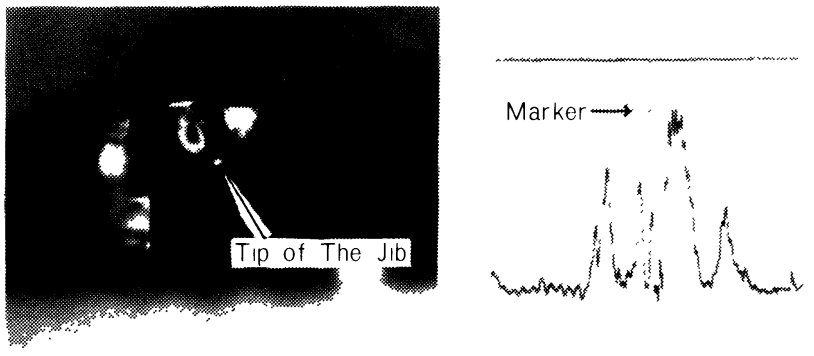

(c) 24 degrees

Fig. 11 Typical photographs of polyester needle threads slipping out of the jib (C type).

ment behavior stable. On the other hand, the turning angle when the needle thread slips out of the bracket was from -4 to 10 degrees with polyester and nylon threads, and from 27 to 33 degrees with cotton threads. The difference between the two thread types was not only big but also scattered widely in the range of 20-30 degrees.

As discussed above polyester and nylon threads behaved quite similarly in disengagement from rotating hook and also in tightening tension. Cotton threads, however distinctively different. In view of this we further compare polyester and cotton threads neglecting nylon threads.

\section{Discussion}

5.1 Mechanism of the Generation of Hook Slipping Tension As discussed above polyester thread and cotton thread behave differently in disengaging from rotating hook. We discuss this point further in detail. 

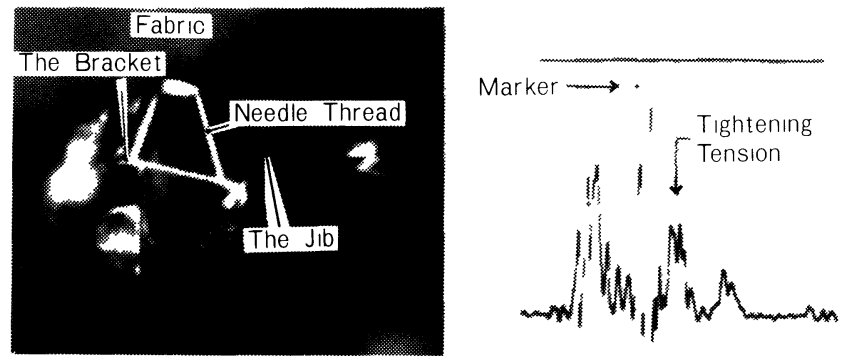

(a) 18 degrees
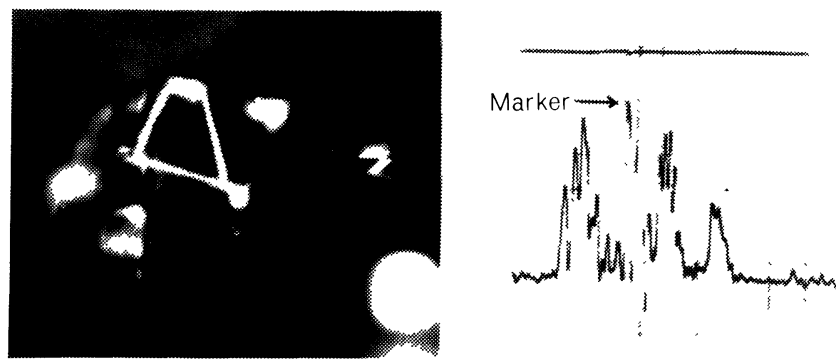

(b) 19 degrees
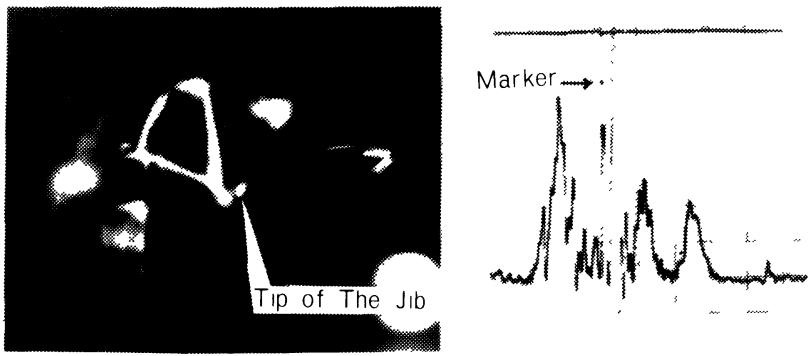

(c) 20 degrees

Fig. 12 Typical photographs of cotton needle threads slipping out of the jib ( $C^{\prime}$ type).

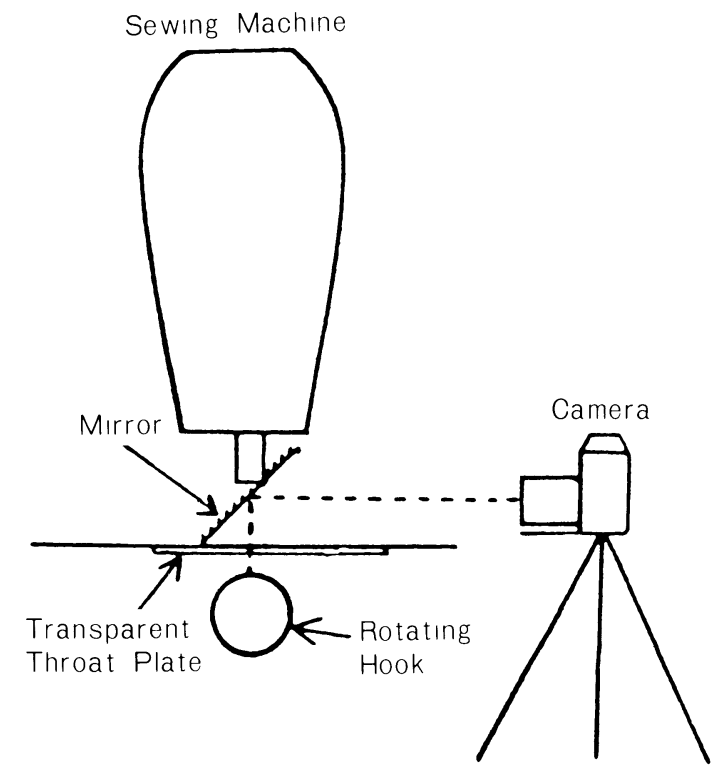

Fig. 13 Schematic of the observation of needle thread in the rotating hook at rest.
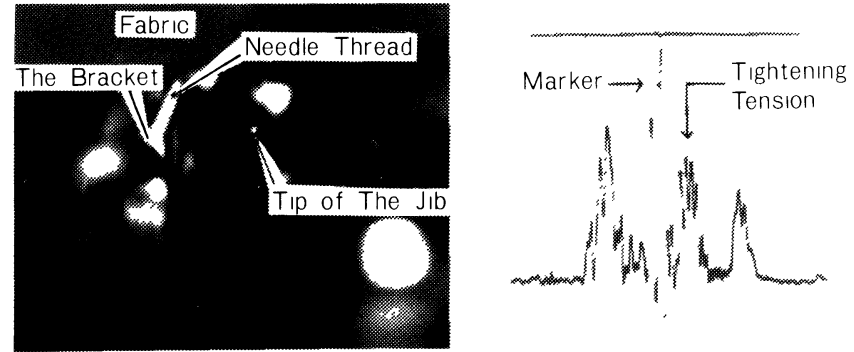

(a) 31 degrees
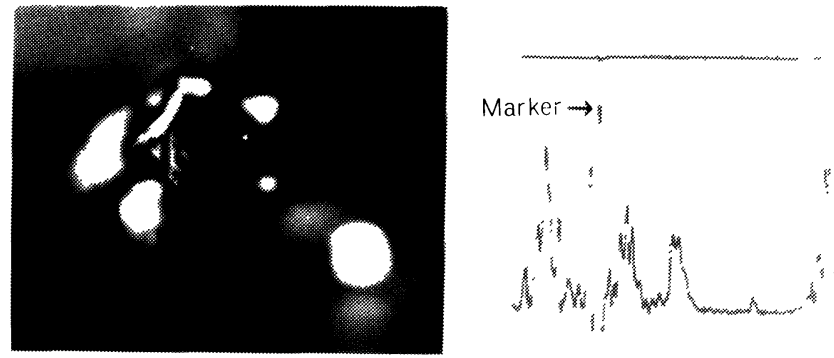

(b) 32 degrees
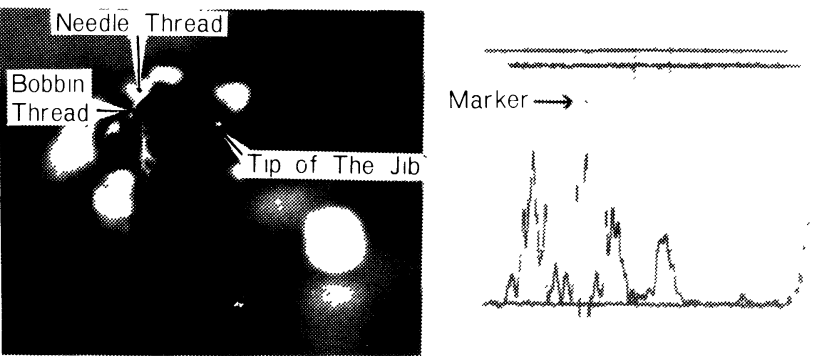

(c) 33 degrees

Fig. 14 Typical photographs of cotton needle threads slipping out of the bracket ( $C^{\prime}$ type).

Fundamentally needle thread tension starts to appear when thread slack is eliminated to become tight. Table 2 shows that in sewing with polyester threads, needle thread is tight in the arm shaft angle range from -4 to 10 degrees. Cotton threads becomes tight at the angle of 19 degrees.

The rotating hook at rest was observed photographically by means of the method illustrated in Figure 14 in order to obtain informations on the relation among the stretched needle thread, the bracket and the jib at the arm shaft angles described above. The throat plate with just needle hole drilled was made of a acrylic plate. The feed dog was removed and the bracket was painted white to facilitate observation. The results obtained are shown photographically in Figure 15. Fig. 15(a) at the arm shaft angle of 9 degrees is equivalent to type $\mathrm{C}$ for polyester thread. The bracket slipping tension in type $\mathrm{C}$ is larger than in other two types. In this case, needle thread slips easily out of the bracket aided by the jib which keep the thread tight. However, when cotton thread slips out of the jib at the arm shaft angle of 


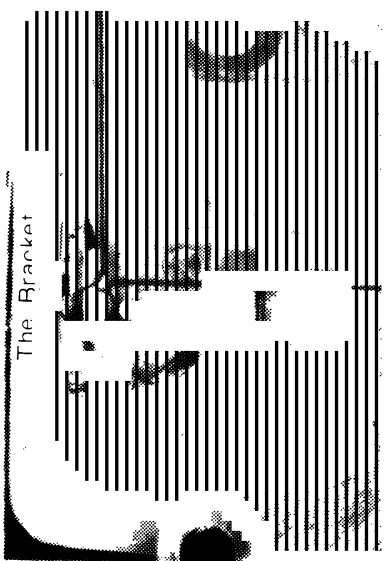

(a) 9 degrees

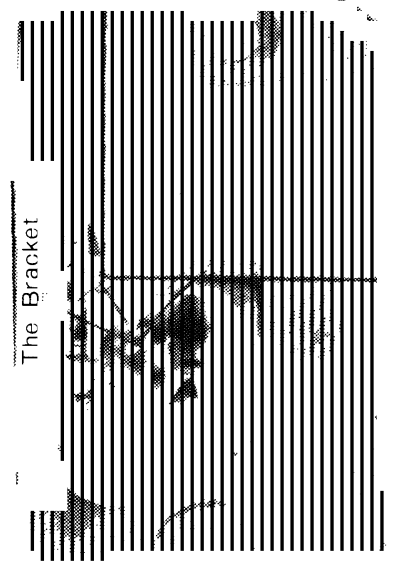

(b) 19 degrees
Fig. 15 Photographs of needle threads due to the set up shown in Fig. 14.

19 degrees as shown in Fig. 15(b), a component of the needle thread tension at the jib aids the needle thread to slip off the jib (the picture does not show the three dimensional configuration of the thread but the state of the thread is such that it easily slips off the jib as soon as a slight increase in tension occurs). And at this time the needle thread is held by the bracket. Therefore, with the cotton threads the needle thread first slips out of the jib and then out of the bracket.

Thus, polyester and cotton threads becoming tight at different arm shaft results in the reversing of the order of their disengagement from the rotating hook.

We further discuss the causes of the difference. Figure 16 shows the behavior of polyester and cotton threads under the same conditions. The polyester thread illustrated in Fig. 16(a) is tight, on the other hand, the cotton thread illustrated in Fig. 16(b) is slack. That means the length of the cotton thread under the throat plate is longer than that of the polyester thread. In order to confirm this observation, needle thread length while in sewing operation was measured. This was done by measuring the needle thread length from the thread take-up spring to the stitch whenever the thread was slack after a sudden stoppage of the machine. The cotton thread had the length of $322+3 \mathrm{~mm}$, and the polyester thread $316+3$, the cotton thread being longer than polyester thread by $6 \mathrm{~mm}$. Clearly the difference is due to the elongational properties of the two sewing threads.

In view of above, polyester thread higher in elongation than cotton thread first slips out of the bracket because the thread becomes tight at a proper arm shaft angle aided by the jib. On the other hand, the cotton thread being longer than polyester thread under the throat plate does not become tight until the arm shaft angle is too big to make the jib unable to assist disengagement resulting in the needle thread first slip out of the jib rather than the bracket.

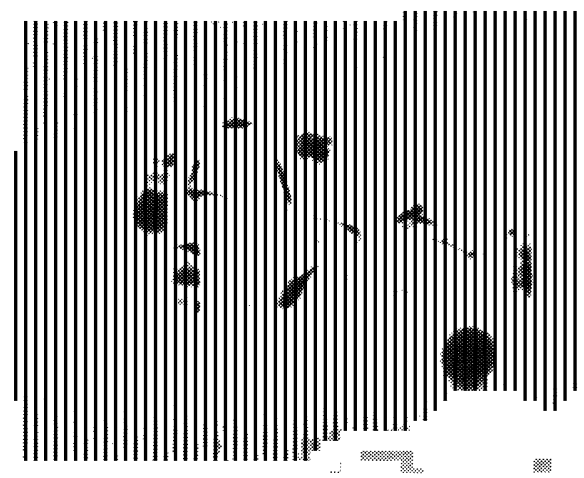

(a) With Polyes ter Filament Thread

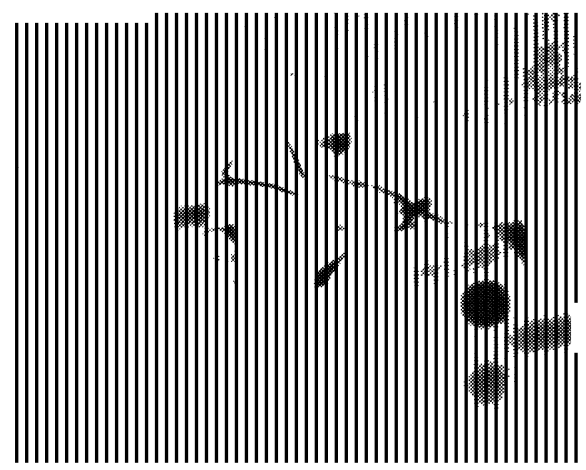

(b) With Cotton Thread

Fig. 16 Difference between polyester and cotton threads at the arm shaft angle of 7 degrees.

Cotton needle threads must become tight at the same arm shaft angle as in polyester threads for the disengagement to be normal.

5.2 Variation of Tightening Tension Caused by Abnormal Disengagement of Needle Threads from Rotating Hook

It was made clear in Section 4.1 that the tightening tension decreased with increasing hook slipping tension in sewing with cotton threads because thread was prematurely drawn out of the tension disc when hook slipping tension was generated before the tightening tension. In view of this needle thread length required when the hook slipping tension generates were investigated with the results shown in Table 3. In the table, the needle thread supply is the slack length of needle thread given by the thread take-up lever, and the needle thread demand is the needle thread length under the fabrics as shown by photographs. With polyester threads, the needle thread supply when the needle thread slips out of the bracket or the jib is larger than the needle thread demand by about $8-9 \mathrm{~mm}$. This excess is absorbed by the thread take-up spring and no thread is drawn out of the tension disc. In case of cotton threads, on the other hand, the difference between the needle thread supply and the needle thread demand is zero. Besides the thread take-up spring is 
Table 3. Comparison between needle thread supply and needle thread demand when needle threads slip out of the hook.

\begin{tabular}{lcccc}
\hline & Polyester & \multicolumn{3}{c}{ Cotton } \\
\hline & $\begin{array}{l}\text { Slipping out } \\
\text { of the bracket }\end{array}$ & $\begin{array}{l}\text { Slipping out } \\
\text { of the jib }\end{array}$ & $\begin{array}{l}\text { Slipping out } \\
\text { of the jib }\end{array}$ & $\begin{array}{l}\text { Slipping out } \\
\text { of the bracket }\end{array}$ \\
\hline $\begin{array}{l}\text { Needle thread behavior* } \\
\text { Turning angle of the arm shaft }\end{array}$ & $(1)$ & $(2)$ & $(1)$ & $(3)$ \\
$\quad$ (degrees) & 10 & 21 & 19 & 30 \\
Needle thread supply (mm) & 45 & 29 & 32 & 19 \\
Needle thread demand (mm) & 37 & 20 & 32 & 19 \\
\hline
\end{tabular}

*Needle thread behavior

(1) (2) (3) Fabrics Needle Thread The Jib Bobbin thread The Bracket

(1)

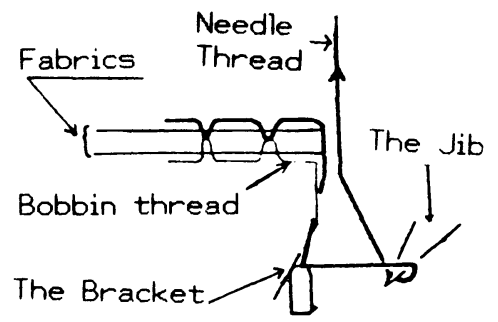

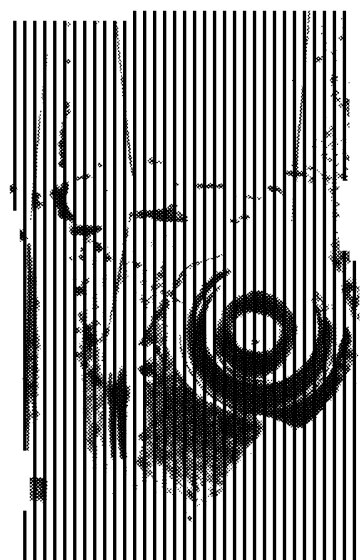

(a) With Polyester Filament Thread (22 degrees)

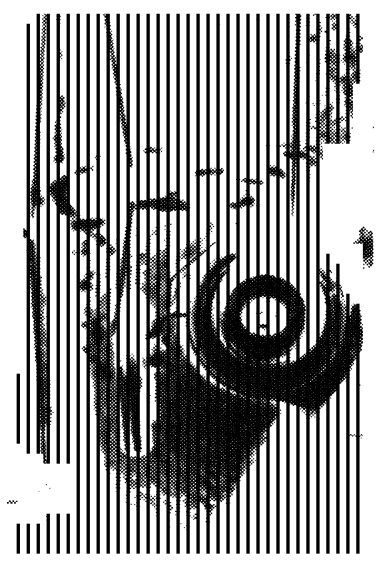

(b) With Cotton Thread (18 degrees)
Fig. 17 Difference in thread take-up spring position under different sewing threads.

close to the maximum position (refer to Fig. 17(b)). Consequently whenever the check spring take up exceeds the allowed limit shortage must be compensated by its elongation or by extra thread drawn out of the tension disc. Points in Fig. 9 were classified by the types in Table 2 to obtain Figure 18. Tightening tension is the smallest in type $C^{\prime}$ having the largest hook slipping tension. In type $C^{\prime}$ thread is estimated to be frequently drawn out of the tension disc to result in
(2)

(3)

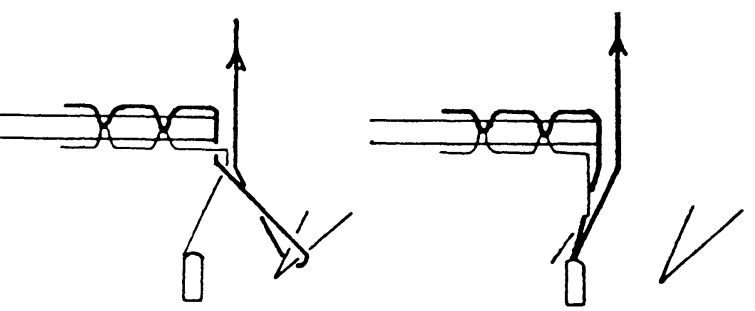


does not affect tightening tension.

\section{Conclusion}

1) Under certain conditions in sewing with cotton threads tightening tension becomes low in level and showed violent variation. In this case the tightening tension was found to decrease with increasing hook slipping tension which is the tension generated when the thread slips out of the jib, or the bracket of the rotating hook. Also the tightening ratio of the seam was higher with cotton threads than with polyester threads.

2) To find out the cause of this abnormality behavior of needle threads as they slipped out of the rotating hook while in sewing operation was photographically observed, and the needle thread tension generated were recorded simultaneously. This revealed that different sewing threads slipped out of the rotating hook in different manner as discussed below.

3) In sewing with cotton threads low in elongation, the rotating hook does not make the needle thread tight until the arm shaft angle becomes too large to cause the needle thread slip out of the jib and out of the bracket thereafter. In other words the jib fails to aid the needle thread first slip out of the bracket.

Consequently when the needle thread disengages from the rotating hook a shortage of thread occures and the shortage cannot be absorbed by the elongation of thread or by the thread take-up spring to result in a drawing out of thread from the tension disc. This in turn causes insufficient tightening tension.

4) In sewing with polyester and nylon threads high in elongation, the needle thread first slips out of the bracket with the help of the jib, and thereafter slips out of the jib. This is the normal disengagement of needle thread from the rotating hook.

\section{Acknowledgements}

We wish to express our thanks to Mr. K. Hara, senior fellow of Technical Research Laboratory, Janome Sewing Machine Co., Ltd., for fruitful discussions. Thanks are also due to Kanagawa Co., Ltd. for supplying the sewing threads used in this work.

\section{References}

[1] R. Miyashita, J. Sekiguchi, M. Sasahara, H. Kandachi; J. Text. Mach. Soc. Japan, 27, P55 (1974).

[2] Y. Ohmura; ibid 21, T211 (1968).

[3] S. Kawanishi, K. Miyamoto, T. Horino; J. Japan Res. Assn. Text. End-Uses, 6, 306 (1965).

[4] N. Ikegami; Tokyo Metropolitan Text. Res. Inst. News, No. 52, p. 2 (1979). 